\title{
Una propuesta de regulación estratégica para el problema de la quema de caña de azúcar en el distrito de La Huaca, Piura, 2015 - 2018.
}

\begin{abstract}
A proposal for strategic regulation for the problem of burning sugarcane in the district of La Huaca, Piura, 2015 - 2018.
\end{abstract}

Recibido: 17 de noviembre de 2019

Aprobado: 18 de junio de 2020

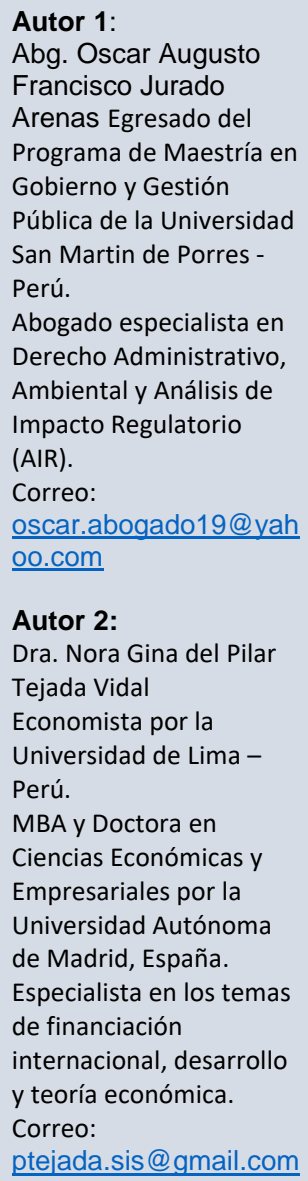

\section{RESUMEN}

Objetivo determinar relación entre la regulación ambiental y la quema de caña de azúcar del distrito de la Huaca, Piura. El presente artículo refleja el desarrollo de una investigación de tipo no experimental y correlacional y está enmarcada en el enfoque mixto. La muestra fue de tipo probabilística que comprende 333 ciudadanos del distrito de La Huaca, Piura. Se utilizó la encuesta para recoger información relevante de los pobladores de La Huaca, así como también se utilizó la recolección de datos mediante el cuestionario y la entrevista a profundidad a 28 expertos en evaluación ambiental respecto de las variables materia de la investigación. Asimismo, para la confiabilidad del instrumento se utilizó el coeficiente de Alfa de Cronbach que salió con alto valor de confiabilidad para las variables de estudio. Los resultados arrojaron que existe relación entre la regulación ambiental y la quema de caña de azúcar del distrito de la Huaca, Piura, teniendo un coeficiente inverso de $(r=-, 207)$ afirmando que la regulación ambiental se relaciona significativamente con la quema de caña de azúcar del distrito de la Huaca, Piura, interpretándose como: $A$ mayor regulación ambiental, entonces menor quema de caña de azúcar. Finalmente, se debe señalar que el término "regulación ambiental" no guarda identidad absoluta a "normas legales", pues es enmarcado en los preceptos de Análisis de Impacto Regulatorio (AIR) el cual propone que la promulgación de normas no es la única manera de regular, pues las autoridades deben tener a su disposición un abanico de alternativas para hacer cambiar la conducta a sujetos regulados y orientarlos a fines públicos relevantes.

Palabras clave: Regulación ambiental, Análisis de Impacto Regulatorio, Quema de Caña de azúcar

\begin{abstract}
Objective of determining what relationship exists between environmental regulation (independent variable) and the burning of sugar cane (dependent variable) in the Huaca district, Piura. This article reflects the development of non-experimental and correlational research and is framed in the mixed approach. The sample was of a probabilistic type comprising 333 citizens of the La Huaca district, Piura. The survey was used to collect relevant
\end{abstract}


information from the residents of La Huaca, as well as data collection using the questionnaire and an in-depth interview with 28 experts in environmental evaluation regarding the variables subject of the investigation. Likewise, for the reliability of the instrument, the Cronbach's alpha coefficient was used, which came out with a high reliability value for the study variables. The results showed that there is a relationship between environmental regulation and the burning of sugarcane in the Huaca district, Piura, having an inverse coefficient of $(r=-, 207)$, affirming that environmental regulation is significantly related to the burning of cane. of sugar from the Huaca district, Piura, interpreted as: The greater the environmental regulation, the less the burning of sugar cane. Finally, it should be pointed out that the term "environmental regulation" does not keep absolute identity to "legal norms", as it is framed in the precepts of Regulatory Impact Assessment (RIA) which proposes that the promulgation of norms is not the only way to regulate, since the authorities must have at their disposal a range of alternatives to change the behavior of regulated subjects and guide them to relevant public purposes.

Key words: Environmental regulation, Regulatory Impact Analysis, Burning of sugar cane

\section{INTRODUCCIÓN}

\section{SITUACIÓN PROBLEMÁTICA}

En el Perú, se cultiva la caña de azúcar específicamente para conseguir 02 productos derivados a saber: el azúcar y el etanol anhidro, siendo este último un biocombustible que se utiliza para rectificar los tradicionales combustibles fósiles y hacerlos más amigables al medio ambiente. Piura es el único departamento del Perú donde se cultiva caña para la producción de etanol con la ejecución del proyecto "Caña Brava" y a partir del 2017 con el proyecto "AgroAurora”.

Las empresas cañeras en el Perú -por lo general- utilizan el método de quema de caña de azúcar para cosecharla, considerando la cantidad de maleza y residuos agrícolas que se genera al cosecharla y además, no existe en la legislación peruana una norma expresa que prohíba la quema de caña. En efecto, por un tema de costos, a las empresas les resulta menos oneroso quemar la caña que contratar trabajadores y maquinarias para cosecharla en verde (cosecha mecanizada en verde). 
El quemado de la caña de azúcar antes y después del corte en la cosecha, es una práctica muy extendida en la eliminación de la cobertura vegetal y control de la maleza que facilitan la preparación y replantación de los suelos con reducción de los costos en la cosecha; a la vez ocasionan la afectación del medio ambiente, la destrucción de materia orgánica y la pérdida de la estructura del suelo con mayor desecamiento y erosión (Aguilar, Pérez, \& Aguilar, 2016, p. 3). Las motivaciones para la quema de caña de azúcar están fundamentadas en criterios científicos verificables y comprobables, pero otras se basan en conceptos subjetivos y presunciones. (Chaves, 2006, pp.248250).

En los ingenios azucareros del Perú, el inconveniente técnico para dejar de quemar y optar por la cosecha mecanizada en verde, es que las máquinas cosechadoras especiales, dejan en el tallo de la caña cortada un residuo vegetal denominado "broza", el cual en caso no se degrade en el suelo, no permitirá que crezca una nueva caña en el tallo, lo que implica a su vez problemas para la siguiente cosecha, llegando incluso a la necesidad de volver a sembrar la caña. Además, el hecho de que la empresa trabaje con máquinas cosechadoras especiales, hace que incurra en costos de mantenimiento y combustibles, los que no incurre al quemar la caña parada. La quema de la caña genera emisión de contaminantes a la atmósfera, principalmente el monóxido de carbono (CO) y material articulado en abundancia (polvo) y esto incide en la calidad de aire y ello a su vez, probablemente en la salud de las personas del distrito de La Huaca que viven en zonas aledañas a los cultivos de caña de azúcar (incremento de riesgo de afectación). La quema de caña constituye entonces una externalidad negativa.

Las externalidades negativas, son efectos económicos colaterales de las acciones de unas personas sobre otras, es decir, que son externos al mercado. Pero, dado que no existen derechos de propiedad sobre una gran cantidad de bienes y que se producen efectos externos al mercado que no son compensados por intercambios mercantiles. 
Por lo tanto, el tratamiento económico de la externalidad se aproxima, al de los bienes públicos (Alva, 2018).

En el caso de las externalidades negativas, se tiene que la acción de un agente privado genera efectos no contratados o sobrecostos a la población (Alva, 2018). En suma, la quema de caña por parte de AGROAURORA y AGRÍCOLA DEL CHIRA constituyen una externalidad negativa que viene siendo asumida por los pobladores de La Huaca como un costo "no contratado previamente", pues los pobladores no "negociaron" con las empresas cañeras si querían o no recibir humo de la quema.

Las empresas AGRíCOLA DEL CHIRA y AGROAURORA, tienen aprobados Estudios de Impacto Ambiental (EIA'S) para el desarrollo de sus actividades agroindustriales por el Ministerio de Agricultura y Riego (MINAGRI) y el Ministerio de la Producción (PRODUCE) respectivamente. Se entendería que estos instrumentos ambientales son la regulación para que las empresas cañeras internalicen la externalidad negativa que producen. Sin embargo, los Estudios de Impacto Ambiental (EIA), no prohíben la quema de caña o de residuos agrícolas, sino establecen un período de adecuación (plazos entre 05 y 30 años) para que las empresas encuentren alternativas ambientalmente viables a la quema de caña. Cabe acotar que, en tanto las empresas cañeras no encuentren muestras reales de la afectación al aire y/o a la salud de las personas por la quema, no tienen los incentivos necesarios para internalizar la externalidad.

Resulta importante señalar, además, que existe un problema de asimetría informativa entre los titulares cañeros y el Estado. Los primeros proponen el cronograma de adecuación de acuerdo a sus "máximos esfuerzos económicos y operativos" posibles; en tanto, el Estado, sin información respecto de las actividades agrícolas, queda sin argumentos para poder reformular u observar con mayor rigurosidad el cronograma propuesto. 
En este punto, es menester reflexionar acerca de la actual regulación para la quema de caña de azúcar. Desde ya se señala que, para efectos del presente trabajo, se entenderá por "regulación” a cualquier actuación estatal (norma que establezca obligaciones, incentivos, acto administrativo, contrato de concesión, etc.) que tenga como finalidad el cambio de conducta de sujetos regulados hacia fines públicos relevantes. (OSINERGMIN, 2016, p.8). Asimismo, bajo el enfoque del AIR, se tiene que es necesario que el Estado asuma esquemas de regulación inteligente, que recurra a técnicas clásicas de regulación estatal (como la prohibición de acciones, multas, etc.) y no estatales (fomento, contratos, etc.) que permita alcanzar el objetivo regulatorio, para adoptar medidas más severas y eficaces (Gunningham y otro, 1998, pp. 381-385).

El presente trabajo se centra en abordar la problemática por la quema de caña de azúcar y residuos agrícolas de los ingenios azucareros en el distrito de La Huaca, provincia de Paita, departamento de Piura, establecer la relación entre la regulación y la quema de caña, y proponer algunas medidas -basadas en un enfoque regulatorio estratégico del AIR- a adoptar para controlar tal externalidad. Por lo tanto, se plantea el siguiente objetivo general. Determinar la relación que existe entre la regulación ambiental y la quema de caña de azúcar del distrito de La Huaca, Piura.

\section{JUSTIFICACIÓN}

El presente artículo se centra en la determinación de la correlación que existe entre la regulación ambiental y la quema de caña de azúcar y su incidencia en la problemática socioambiental en el distrito de La Huaca.

Una vez determinada la correlación, se realizan las recomendaciones pertinentes orientadas a las acciones que tendrían que adoptar las entidades públicas para buscar la solución a esta problemática, considerando un diseño de regulación estratégica de acuerdo a los preceptos del AIR. 
La línea de investigación del presente artículo es correspondiente a la de políticas públicas, pues se propone a una acción estatal para atender una externalidad negativa que se ha convertido en problema público.

\section{ANTECEDENTES}

En el Perú no existe un estudio epidemiológico oficial, desarrollado por el Ministerio de Salud (MINSA) que de cuenta sobre el impacto de la quema de caña de azúcar en la salud de las personas.

No obstante, existe a nivel nacional estudios sobre la incidencia negativa de la quema de caña de azúcar en el componente aire, como el de Villalobos (2017) en su estudio "Influencia de la quema de biomasa de caña de azúcar en la concentración de PM2.5 en el aire de la zona urbana de Laredo", tesis para optar el titulo de ingeniero ambiental.

Por otro lado, no puede dejarse de mencionar el trabajo de López y otros (2017) en su investigación titulada "Diagnóstico Operativo Empresarial (DOE), de Agrícola del Chira S.A., tesis para obtener el grado de magíster en administración estratégica", el cual aborda la problemática que la genera a la empresa AGRíCOLA DEL CHIRA la quema de la broza de caña y propone enterrarla, a efectos de mejorar la gestión económica empresarial.

\section{MARCO NORMATIVO AMBIENTAL APLICABLE A LAS ACTIVIDADES DE}

\section{CULTIVO DE CAÑA DE AZÚCAR.}

Como se mencionó en la introducción, en el Perú no existe normativa específica que regule la gestión ambiental de la quema de caña de azúcar, y por ende no existen obligaciones ambientales fiscalizables específicas para las empresas cañeras. Sin embargo, es menester repasar algunas normas de carácter ambiental en la normativa nacional que incidirían de alguna u otra forma en la quema de caña de azúcar. 
Una propuesta de regulación estratégica para el problema de la quema de caña de azúcar en el distrito de La Huaca, Piura, 2015 - 2018.

- Ley del Sistema Nacional de Evaluación del Impacto Ambiental (Ley del SEIA), Ley № 27446 y su Reglamento aprobado por Decreto Supremo № 019-2009-MINAM

Este es el marco normativo transectorial ambiental, que regula la obligatoriedad de que toda actividad de cualquier sector económico (minería, transportes, industria, agricultura, electricidad, hidrocarburos, etc.) que genere impactos al ambiente, debe contar con un Estudio de Impacto Ambiental (EIA) de manera previa al inicio de obras ${ }^{1}$.

Asimismo, se regulan los procedimientos a seguir para que los titulares de proyectos obtengan la aprobación de su respectivo EIA, así como también, se establece la obligación de que los titulares, a lo largo de la vida útil de su proyecto, ejecuten una Estrategia de Manejo Ambiental (EMA) la cual debe contener medidas socioambientales.

Respecto de este marco normativo, resulta aplicable a las empresas cañeras la obligatoriedad de contar con un EIA aprobado por la respectiva autoridad ambiental competente para sus actividades. Sin embargo, al ser normativa de carácter general, no establece obligaciones específicas o un quantum mínimo que debería tener en cuenta las empresas cañeras para la actividad de quema.

- Ley del Sistema Nacional de Evaluación y Fiscalización Ambiental, Ley № 29325 (Ley del SINEFA)

Después de la aprobación del EIA, se necesita un sistema del Estado para que las obligaciones ambientales que haya asumido el Titular se cumplan. De ahí, es que nace el sistema de fiscalización ambiental, identificando al Organismo de Evaluación y Fiscalización Ambiental (OEFA) como ente rector del mismo.

\footnotetext{
${ }^{1}$ Ley del Sistema Nacional de Evaluación del Impacto Ambiental (Ley del SEIA), Ley № 27446 “Artículo 3.Obligatoriedad de la certificación ambiental No podrá iniciarse la ejecución de proyectos ni actividades de servicios y comercio referidos en el artículo 2 y ninguna autoridad nacional, sectorial, regional o local podrá aprobarlas, autorizarlas, permitirlas, concederlas o habilitarlas si no cuentan previamente con la certificación ambiental contenida en la Resolución expedida por la respectiva autoridad competente."
} 
El sistema de fiscalización ambiental peruano, básicamente, se centra en acciones de supervisión, esquema de multas ante incumplimientos al EIA y/o a la norma y medidas administrativas (suspensión, cierre de actividades riesgosas, etc.). El artículo 11 de la Ley del SINEFA señala:

\section{"Artículo 11.- Funciones generales}

Función fiscalizadora y sancionadora: comprende la facultad de investigar la comisión de posibles infracciones administrativas sancionables y la de imponer sanciones por el incumplimiento de obligaciones y compromisos derivados de los instrumentos de gestión ambiental, de las normas ambientales, compromisos ambientales de contratos de concesión y de los mandatos o disposiciones emitidos por el OEFA, en concordancia con lo establecido en el artículo 17. Adicionalmente, comprende la facultad de dictar medidas cautelares y correctivas".

En ese sentido, el OEFA fiscalizará que las empresas cañeras de La Huaca cumplan con los programas de "quema contralada" aprobados en los EIA'S por PRODUCE y MINAGRI.

No obstante, de acuerdo a la normativa, el OEFA, eventualmente, podría ordenar medidas administrativas de cese o reducción de quema a las empresas cañeras en La Huaca, en tanto si se demuestra técnicamente que la manera como el PRODUCE o el MINAGRI aprobó el EIA constituye un riesgo de afectación al medio ambiente o salud de las personas. Sin embargo, el OEFA (al igual que el PRODUCE y MINAGRI) carece de información para poder regular la actividad cañera mediante medidas administrativas o el costo para obtener esta información es muy alto.

- Reglamento de Manejo de los Residuos Sólidos del Sector Agrario, aprobado por Decreto Supremo № 016-2012-AG.

Quizá esta norma se aproxima a ser una regulación a las empresas cañeras. En el artículo 27, se señala lo siguiente: 
Una propuesta de regulación estratégica para el problema de la quema de caña de azúcar en el distrito de La Huaca, Piura, 2015 - 2018.

\section{"Artículo 27.- Gestión de los residuos de las actividades agrícolas}

27.1 Los restos vegetales de cultivos o cosecha, pueden ser reaprovechados como forrajes de animales de crianza; así también se puede realizar su aplicación directa en la superficie del suelo, para incrementar el nivel de fertilidad, favorecer la estructura y textura del suelo y con el tiempo incrementar la infiltración del agua y reducir la erosión eólica e hídrica.

El compostaje es una opción de valorización para los residuos agrícolas donde estos restos vegetales se usan como estructurantes de aporte de carbono, para el buen funcionamiento del proceso de compostaje, también puede usarse como biocombustibles

Está prohibido realizar la quema de dichos residuos vegetales" (énfasis agregado).

Bajo este artículo, resulta válido preguntarse ¿Entonces la quema de caña sí se encuentra prohibida por norma? Al respecto, es necesario realizar las siguientes precisiones:

a) La prohibición está referida a residuos sólidos; en tanto, la caña de azúcar,

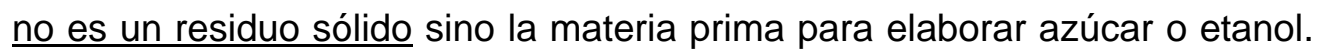
Las razones por las que se quema caña, se explicaron en la introducción del presente artículo.

b) En ese sentido, de acuerdo al precepto jurídico "las normas que restringen derechos o establecen prohibiciones, deben interpretarse de manera restrictiva"2, no se podría concluir que por interpretación extensiva o analógica que el Decreto Supremo № 016-2012-AG, prohíbe también la quema de caña de azúcar. Aunado a ello, ninguna autoridad ambiental a nivel nacional ha 
interpretado de manera extensiva esta norma, es más, existen proyectos normativos en el congreso relacionados a la reducción y/o prohibición expresa de la quema de caña, lo que refleja la necesidad de promulgación de normativa específica prohibitiva

Está prohibido realizar la quema de dichos residuos vegetales" (énfasis agregado).

Bajo este artículo, resulta válido preguntarse ¿Entonces la quema de caña sí se encuentra prohibida por norma? Al respecto, es necesario realizar las siguientes precisiones:

a) La prohibición está referida a residuos sólidos; en tanto, la caña de azúcar,

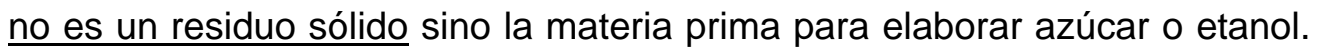
Las razones por las que se quema caña, se explicaron en la introducción del presente artículo.

b) En ese sentido, de acuerdo al precepto jurídico "las normas que restringen derechos o establecen prohibiciones, deben interpretarse de manera restrictiva"3, no se podría concluir que por interpretación extensiva o analógica que el Decreto Supremo № 016-2012-AG, prohíbe también la quema de caña de azúcar. Aunado a ello, ninguna autoridad ambiental a nivel nacional ha interpretado de manera extensiva esta norma, es más, existen proyectos normativos en el congreso relacionados a la reducción y/o prohibición expresa de la quema de caña, lo que refleja la necesidad de promulgación de normativa específica prohibitiva.

c) No obstante, en caso las industrias cañeras ejecuten cosecha mecanizada en verde y se genere broza, este último sí constituye un residuo agrícola y por

${ }^{3}$ El Tribunal Constitucional peruano lo considera un principio general del derecho. Véase la STC recaída en el Exp. $\mathrm{N}^{\circ}$ 01385-2010-PA/TC 
ende, no puede ser quemado. A pesar de ello, la Dirección General de Asuntos Ambientales Agrarios (DGAAA) del MINAGRI, en el año 2016, aprobó un estudio ambiental a la AGRíCOLA DEL CHIRA, permitiéndole ejecutar quemas de broza de caña por un plazo máximo de 05 años y vencido este, la empresa ya debe haber implementado un sistema para reutilizar, valorizar o disponer de manera ambientalmente amigable la broza de caña. Cabe acotar, que se prohibió la quema de caña parada.

d) Como no podía ser de otra manera, el pronunciamiento de la DGAAA, resulta ser bastante cuestionable, pues habría sido expedido en contra de una norma prohibitiva expresa. Sin embargo, la DGAAA fundamentó su pronunciamiento en una opinión técnica brindada por el Servicio Nacional de Sanidad Agraria (SENASA) durante la evaluación del estudio ambiental de AGRÍCOLA DEL CHIRA. La opinión técnica brindada por SENASA se sustenta en que es necesario "eliminar" residuos agrícolas a efectos de que estos no generen la aparición de plagas o vectores (roedores). De esta manera, las quemas autorizadas por la DGAAA son para evitar que en el distrito de La Huaca aparezcan plagas y vectores que pongan en peligro la salud ambiental. Finalizando, queda hasta la fecha la pregunta en el aire ¿debemos entender por "eliminar" el residuo, como quemarlo?

e) Finalmente, la Dirección General de Asuntos Ambientales de Industria (DGAAMI) del PRODUCE, aprobó el EIA a favor de AGROAURORA, permitiendo la quema de caña (30 años), pero respetando la normativa en comento, esto es, prohibió de manera expresa que se quemara broza que se pudiese generar y estableció que esta sea apilada y en 05 años, al igual que AGRÍCOLA DEL CHIRA, se encuentre un mecanismo alternativo para disponerla. Cabe señalar, que durante la evaluación del EIA, el PRODUCE no solicitó opinión técnica al SENASA 


\section{- Reglamento de Gestión Ambiental para la Industria Manufacturera y} Comercio Interno aprobado por Decreto Supremo № 017-2015-PRODUCE y Reglamento de Gestión Ambiental del Sector Agrario, aprobado por Decreto Supremo № 019-2012-AG.

Los Reglamentos en mención, corresponden a los sectores industrial (PRODUCE) y agrícola (MINAGRI). En ellos se establecen obligaciones ambientales generales que deben seguir los titulares en el desarrollo de sus actividades (adecuado manejo de residuos sólidos, de emisiones, efluentes que genere la actividad, etc). Sin embargo, en ninguno se establece una obligación específica para la industria cañera.

Téngase en cuenta que estos Reglamentos establecen obligaciones dirigidas a actividades económicas diversas bajo su competencia. Por ejemplo, en el sector industria, las obligaciones ambientales del Reglamento deben ser cumplidas por titulares de actividades cementeras, curtiembres, agroindustriales, industrias alimentarias, etc.; en tanto, en el sector Agrario, las obligaciones ambientales deben ser cumplidas por titulares de cultivos diversos, ganaderos e incluso de proyectos de irrigaciones. Esa es la razón, por la cual, tales obligaciones son diseñadas de manera ciertamente generalorma y modo de cálculo de los derechos de pesca del recurso anchoveta, ha sufrido cambios a lo largo del tiempo. Tradicionalmente, el derecho de pesca de este recurso, se estableció como un porcentaje de la Unidad Impositiva Tributaria (UIT), pero a partir del año 2006 empezó a aplicarse el valor FOB de la harina de pescado como unidad referencial, debido a la tendencia creciente del precio mundial de la harina de pescado, producto principal del procesamiento de este pequeño recurso pelágico. El derecho de pesca es un concepto asociado directamente con la actividad extractiva; sin embargo, como podrá advertirse, ni la UIT ni el valor FOB, tienen vinculación directa con la actividad extractiva del recurso. 
Una propuesta de regulación estratégica para el problema de la quema de caña de azúcar en el distrito de La Huaca, Piura, 2015 - 2018.

Asimismo, estos reglamentos establecen los procedimientos sectoriales a seguir por parte del PRODUCE y MINAGRI para la aprobación de los respectivos EIA'S.

Cabe señalar que, en la actualidad, el MINAGRI aprueba el EIA, solo si el titular propone únicamente cultivo de caña. En tanto, PRODUCE aprobará el EIA si el titular propone el proyecto conjunto de cultivo de caña y producción de azúcar o únicamente la producción de azúcar.

- $\quad$ Ley Orgánica de Municipalidades (LOM), Ley № 27972.

De una primera lectura a este cuerpo normativo, pareciera que tanto las Municipalidades Provinciales y Distritales tuvieran una incidencia directa en la regulación de la quema de caña. A continuación, se cita el artículo pertinente:

\section{“ARTÍCULO 80.- SANEAMIENTO, SALUBRIDAD Y SALUD}

Las municipalidades, en materia de saneamiento, salubridad y salud, ejercen las siguientes funciones:

1. Funciones específicas exclusivas de las municipalidades provinciales:

2. Regular y controlar la emisión de humos, gases, ruidos y demás elementos contaminantes de la atmósfera y el ambiente.

3. Funciones específicas exclusivas de las municipalidades distritales:

4. Fiscalizar y realizar labores de control respecto de la emisión de humos, gases, ruidos y demás elementos contaminantes de la atmósfera y el ambiente" (énfasis agregado).

El lector de este artículo lo enfocaría al caso de la quema de caña en el distrito de La Huaca y pensaría que la Municipalidad correspondiente podría expedir una Ordenanza Municipal prohibiendo la quema de caña, aplicando incluso sanciones.

\footnotetext{
1 En ese sentido, véase la doctrina jurisprudencial de "test de competencia" desarrollada por el Tribunal Constitucional (STC № 0020 y 0021-2005-PI/TC, fundamentos 32 a 79.
} 
Sin embargo, el escenario antes descrito podría conllevar a que en la práctica exista un eventual conflicto de competencias entre el PRODUCE y/o MINAGRI, que regula la quema de caña de azúcar mediante EIA’S y la Municipalidad que emita Ordenanzas y actos municipales derivados de estas.

Al respecto, de acuerdo a la reiterada jurisprudencia del Tribunal Constitucional peruano, ante aparentes controversias o conflictos de competencias entre distintos niveles de gobierno, se debe aplicar un enfoque de complementariedad, armonización, priorizando o atribuyendo la competencia a la entidad de gobierno central, según corresponda.

En ese sentido, los EIA'S de las empresas cañeras son aprobados en el marco de competencias en materia ambiental de las entidades del Gobierno Central (PRODUCE o MINAGRI) atribuidas por la Ley del SEIA, por lo que una Ordenanza Municipal prohibiendo la quema de caña (aspecto que es regulado por PRODUCE o MINAGRI) sería cuestionable.

Resulta importante señalar además, que en tanto, la LOM atribuye a las Municipalidades una competencia "macro" o "general" de regulación de "humos y gases" (pudiendo regularse incluso, las chimeneas de las viviendas), las normas ambientales son específicas para la gestión ambiental de determinado sector.

En todo caso, a efectos de no vaciar de contenido a la competencia municipal en comento, las Municipalidades podrían regular de manera "indirecta" a la industria cañera. Por ejemplo, expedir una Ordenanza que multe a la industria cañera por ensuciar la vía pública con ceniza de quema de caña. Este tipo de Ordenanza sí sería compatible con lo aprobado en el EIA de las empresas cañeras. 
Una propuesta de regulación estratégica para el problema de la quema de caña de azúcar en el distrito de La Huaca, Piura, 2015 - 2018.

\section{DESARROLLO}

\section{Metodología}

La presente investigación utiliza el enfoque mixto, según Hernandez, Fernandez, \& Baptista (2014) porque se utilizó las fortalezas y bondades del enfoque cuantitativo (CUAN) y del enfoque cualitativo (CUAL), culminando con una integración de los mencionados enfoques para así tener una mayor comprensión del problema de estudio (p. 580). El presente artículo tiene un diseño no experimental y de tipo correlacional. Las correlaciones pueden ser positivas (directamente proporcionales) 0 , negativas (inversamente proporcionales).

Si es positiva, significa que los casos que muestren altos valores en una variable tenderán también a manifestar valores elevados en la otra variable. Si es negativa implica que casos con valores elevados en una variable tenderán a mostrar valores bajos en la otra variable (Hernández \& Mendoza, 2018,p. 110). De acuerdo a Hernández et al. (2017) "los estudios correlacionales es el grado de relación entre las variables, miden cada una y posteriormente cuantifican y analiza la asociación" (p. 779).

En la presente investigación, la población será tomada de los jefes de hogar del distrito de la Huaca, el cual tiene 2608 (2 134 varones y 474 mujeres) según (INEI, 2007) a los cuales se les aplicó el instrumento de medición denominado cuestionario. La presente investigación, tiene una muestra de 333 ciudadanos del distrito de La Huaca a los cuales se les aplicará el instrumento de medición denominado cuestionario, la muestra según, Sánchez (2010). Para el presente artículo, se aplicó un cuestionario a 333 ciudadanos del distrito de La Huaca respecto de cada variable que se pretende probar su correlación (regulación ambiental y quema de caña). Según Hernández et al. (2014) en la presente investigación desde la parte cuantitativa se utilizó como instrumento de medición el cuestionario que viene a ser "Conjunto de preguntas respecto de una o más variables que se van a medir" (p. 217). 
Desde el lado cualitativo, se utilizó la entrevista, la cual, según Hernández et al. (2014) se define como una reunión para conversar e intercambiar información entre una persona (el entrevistador) y otra (el entrevistado) u otras (entrevistados). La entrevista a profundidad se aplicó a 28 expertos, de los cuales destacan especialistas en evaluación y fiscalización ambiental del sector público (MINAGRI, PRODUCE y OEFA), así como también a representantes de empresas cañeras.

Tabla 1

\section{Especialistas en regulación ambiental y cultivo de caña de azúcar entrevistados}

\begin{tabular}{|c|c|c|}
\hline Experto & Profesión & Experiencia relacionada al tema de tesis \\
\hline Ada Alegre Chang & Abogada & $\begin{array}{l}\text { Abogada experta en temas de gestión } \\
\text { ambiental. Gerente de la consultora legal } \\
\text { ambiental "Ada Alegre Consultores" }\end{array}$ \\
\hline Cecilia Torre Sandoval & Abogada & $\begin{array}{c}\text { Directora de la Dirección de Gestión } \\
\text { Ambiental del PRODUCE. }\end{array}$ \\
\hline Paulo Delgado Neyra & Abogado & $\begin{array}{c}\text { Director de Evaluación Ambiental de } \\
\text { PRODUCE }\end{array}$ \\
\hline Javier Taype Yauri & Ingeniero Agrónomo & Evaluador Ambiental PRODUCE \\
\hline $\begin{array}{l}\text { Miguel Carranza } \\
\text { Palomares }\end{array}$ & Ingeniero Ambiental & Evaluador Ambiental PRODUCE \\
\hline $\begin{array}{l}\text { Ricardo Cevallos } \\
\text { Morales }\end{array}$ & Ingeniero Industrial & Evaluador Ambiental PRODUCE \\
\hline Gerardo Muñoz Guerra & Ingeniero Geográfo & Evaluador Ambiental PRODUCE \\
\hline Richard Alca Ayaque & Ingeniero Geográfo & Evaluador Ambiental PRODUCE \\
\hline $\begin{array}{l}\text { Gatsby Ampuero } \\
\text { Armanza }\end{array}$ & Ingeniero Agrónomo & $\begin{array}{c}\text { Director de Gestión Ambiental del } \\
\text { MINAGRI }\end{array}$ \\
\hline Franco Santillán Illesca & Sociólogo & $\begin{array}{l}\text { Experto en temas socioambientales de la } \\
\text { Dirección de Evaluación Ambiental de } \\
\text { PRODUCE. }\end{array}$ \\
\hline Jorge Alva Pasapera & Abogado & $\begin{array}{l}\text { Coordinador de Supervisión Ambiental } \\
\text { de Industria del OEFA }\end{array}$ \\
\hline Dixon Pinedo Ordoñez & Biólogo & $\begin{array}{l}\text { Supervisor de la Coordinación de } \\
\text { Supervisión Ambiental de Agricultura del } \\
\text { OEFA }\end{array}$ \\
\hline $\begin{array}{l}\text { Mary Ann Huancayo } \\
\text { Flores }\end{array}$ & Ingeniera Ambiental & $\begin{array}{l}\text { Supervisor de la Coordinación de } \\
\text { Supervisión Ambiental de Industria del } \\
\text { OEFA }\end{array}$ \\
\hline Hugo Cevallos Morales & Ingeniero Industrial & $\begin{array}{l}\text { Supervisor de la Coordinación de } \\
\text { Supervisión Ambiental de Industria del } \\
\text { OEFA }\end{array}$ \\
\hline $\begin{array}{l}\text { Giuliana Tiravanti } \\
\text { Beoutis }\end{array}$ & $\begin{array}{l}\text { Licenciada en } \\
\text { Química }\end{array}$ & $\begin{array}{l}\text { Supervisor de la Coordinación de } \\
\text { Supervisión Ambiental de Industria del } \\
\text { OEFA }\end{array}$ \\
\hline
\end{tabular}


Una propuesta de regulación estratégica para el problema de la quema de caña de azúcar en el distrito de La Huaca, Piura, 2015 - 2018.

\begin{tabular}{|c|c|c|}
\hline Carlos Ortiz Rojas & Ingeniero Ambiental & $\begin{array}{l}\text { Supervisor de la Coordinación de } \\
\text { Supervisión Ambiental de Industria del } \\
\text { OEFA }\end{array}$ \\
\hline María Alemán Peralta & Ingeniera Forestal & $\begin{array}{l}\text { Supervisor de la Coordinación de } \\
\text { Supervisión Ambiental de Industria del } \\
\text { OEFA }\end{array}$ \\
\hline Álvaro Martínez Vila & Ingeniero Ambiental & $\begin{array}{l}\text { Supervisor de la Coordinación de } \\
\text { Supervisión Ambiental de Industria del } \\
\text { OEFA }\end{array}$ \\
\hline $\begin{array}{l}\text { Eduardo Melgar } \\
\text { Córdova }\end{array}$ & Abogado & $\begin{array}{l}\text { Ex Director de Fiscalización y Aplicación } \\
\text { de Incentivos (DFAI) del OEFA }\end{array}$ \\
\hline Ronald Ordaya Pando & Ingeniero Mecánico & $\begin{array}{l}\text { Ex Director de Evaluación Ambiental de } \\
\text { PRODUCE }\end{array}$ \\
\hline Efraín Salas Valverde & Ingeniero Mecánico & $\begin{array}{l}\text { Gerente de Proyectos de la empresa } \\
\text { azucarera AGRO INDUSTRIAL } \\
\text { PARAMONGA S.A.A. }\end{array}$ \\
\hline $\begin{array}{l}\text { Welser Cabrera } \\
\text { Méndez }\end{array}$ & Ingeniero Ambiental & $\begin{array}{l}\text { Jefe del Área de Medio Ambiente de la } \\
\text { empresa azucarera AGRO INDUSTRIAL } \\
\text { LAREDO S.A.A. }\end{array}$ \\
\hline $\begin{array}{l}\text { José Antonio Mosquera } \\
\text { Chalán }\end{array}$ & Ingeniero Industrial & $\begin{array}{l}\text { Jefe del Área de Medio Ambiente de la } \\
\text { EMPRESA AGROINDUSTRIAL } \\
\text { POMALCA S.A.A. }\end{array}$ \\
\hline Julio Talledo De Lama & Abogado & $\begin{array}{l}\text { Asesor Legal de las empresas } \\
\text { azucareras AGROAURORA S.A.C. y } \\
\text { AGROLMOS S.A.A. }\end{array}$ \\
\hline $\begin{array}{l}\text { Vanessa Hurtado } \\
\text { Carrión }\end{array}$ & Ingeniera Ambiental & $\begin{array}{l}\text { Ingeniera del área de medio ambiente de } \\
\text { la empresa AGRÍCOLA DEL CHIRA } \\
\text { S.A.C. }\end{array}$ \\
\hline Luciano Trelles Flores & $\begin{array}{l}\text { Ingeniero } \\
\text { Electrónico }\end{array}$ & $\begin{array}{l}\text { Regidor de la Municipalidad Distrital de } \\
\text { La Huaca, período } 2015-2018\end{array}$ \\
\hline $\begin{array}{l}\text { Carlos Salazar } \\
\text { Meléndez }\end{array}$ & Médico & Jefe del Centro de Salud de La Huaca \\
\hline Edgard Aliaga Lártiga & Ingeniero Industrial & $\begin{array}{l}\text { Gerente de Operaciones Agrícolas de la } \\
\text { empresa STEVIA ONE PERÚ S.A.C. }\end{array}$ \\
\hline
\end{tabular}

Elaboración : Propia

\section{ANÁLISIS Y DISCUSIÓN DE RESULTADOS}

Los resultados cuantitativos obtenidos en esta investigación condujeron en términos generales a establecer que no existe correlación significativa entre la regulación ambiental y la quema de caña de azúcar del distrito de la Huaca, Piura, período 2015 2018. Un 39 \% (130 personas) está de acuerdo que exista una regulación ambiental en relación al quemado de caña de azúcar. En tanto, un 83.2\% (277 personas), indican que el quemado de caña de azúcar afecta la salud de las personas causando enfermedades respiratorias como el asma bronquial. Asimismo, contamina el suelo. 


\section{Interpretación}

Se advierte que la significancia obtenida fue menor que el nivel propuesto $(p=0,000>0,05)$, por lo que se rechaza la hipótesis nula, consecuentemente se tiene que: La regulación ambiental se relaciona significativamente con la quema de caña de azúcar del distrito de la Huaca, Piura, periodo, 2015-2018.

Teniendo un coeficiente inverso de $(r=-, 207)$, interpretándose como: A mayor regulación ambiental, entonces menor quemado de caña de azúcar.

En tanto, los resultados cualitativos, arrojan que es necesaria una regulación ambiental para reducir la quema de caña o de broza, al ser esta una actividad riesgosa. Esta conclusión general coincide con lo señalado en la parte de análisis cuantitativo: $A$ mayor regulación, menor quema de caña.

No obstante, se debe precisar que, de acuerdo a las directrices y conceptos del Análisis de Impacto Regulatorio, (AIR) citado en las bases teóricas de la referida investigación, el tipo de regulación para reducir la quema de caña de azúcar estaría enfocada al marco de promoción e incentivos para la gestión de la broza de caña (residuo sólido vegetal), antes que una regulación que establezca obligaciones y sanciones.

El esquema regulatorio "prohibición - sanción", se pronostica que sería ineficaz o en todo caso incompleto, para desincentivar la quema de la caña, pues, siguiendo lo señalado por el experto Dixon Pinedo y los demás supervisores del Organismo de Evaluación y Fiscalización Ambiental (OEFA), se necesitaría encontrar a la empresa azucarera "con las manos en la masa" quemando caña de azúcar o broza, por su personal incumpliendo lo aprobado en el EIA o norma respectiva, para poder eventualmente multarla.

Esta situación es prácticamente imposible, pues cuando llegan los supervisores del OEFA a atender denuncias por quema de caña, el evento ya finalizó y las empresas lo 
Una propuesta de regulación estratégica para el problema de la quema de caña de azúcar en el distrito de La Huaca, Piura, 2015 - 2018.

reportan como hecho fortuito $u$ originado por terceros ajenos al ingenio y no existe prueba idónea que desvirtúe lo afirmado por la empresa. Esta situación ya se da en la práctica y se seguiría dando en caso se promulgue una norma prohibiendo la quema de caña.

En suma, así se establezca prohibiciones legales de no quemar caña, el enforcement, la actividad de fiscalización ambiental afronta complicaciones para hacer cumplir la norma. Aunado a ello, el esquema de multas, cuando se aplica efectivamente pierde su carácter disuasivo, se desnaturaliza, pues el infractor de alguna manera paga el costo por incumplir la norma. Por otro lado, de acuerdo a lo señalado por los expertos (Gatsby Ampuero, Richard Alca, Paulo Delgado, Efraín Salas, Welser Cabrera y Javier Taype principalmente), se tiene que a la fecha ya existen ingenios azucareros que tienen máquinas cosechadoras especiales que aplican parcialmente a sus campos de cultivo de caña, pero no la aplican en un $100 \%$, debido a la cantidad de broza que se genera sin degradarse en el suelo y no saben qué hacer o como disponerla, entendiéndose esto como un gasto mayúsculo a asumir. Ante esto, se opta por quemar la caña y desparecer el residuo vegetal antes que se genere.

Siguiendo lo señalado por los expertos, AGRO INDUSTRIAL LAREDO S.A.A., AGRO INDUSTRIAL PARAMONGA S.A.A. y las mismas AGRÍCOLA DEL CHIRA S.A.C. y AGROAURORA S.A.C., son un claro ejemplo de ingenios que tienen este problema de la broza de caña y de ahí la quema de caña de azúcar, sin dejar de mencionar que LAREDO ya cuenta con un proyecto piloto de planta para elaborar compostaje, donde se utiliza la broza como materia prima, siendo que este proyecto piloto no soporta la cantidad de broza que se genera en este ingenio. Asimismo, LAREDO viene realizando estudios técnicos a fin de determinar si la broza puede servir como combustible en los calderos de su planta industrial.

Por otra parte, se destaca el caso del ingenio azucarero AGROLMOS S.A.A., que no necesitó ningún tipo de incentivo tributario o subsidio del Estado para dejar de quemar 
caña parada, a pesar de que su EIA le permite quemar caña sin ningún tipo de restricción o control. No obstante, si bien en este ingenio la broza logra degradarse en el suelo, sería más eficiente y correspondiente a los preceptos de la economía circular, que este residuo vegetal se valorice, considerando, además, los problemas de plagas que tiene este ingenio para lograr que la broza se degrade.

En este contexto, si por ejemplo, el Estado peruano optara por el fomento de instalación de plantas de valorización de residuos (para convertirlos en compost, en energía eléctrica o en biogás), más bien, la broza de caña ya no sería un "problema" para las empresas azucareras, sino esta sería materia prima para las plantas de valorización, incluso, el titular de las plantas de valorización pagaría a los ingenios por obtener broza de caña.

Es importante señalar, que el titular del ingenio azucarero podría también implementar proyectos para valorizar la broza. No obstante, sería más eficiente que el Estado gestione esto, pues daría a su vez solución a los botaderos municipales; en tanto las empresas cañeras, se enfocarían en su productividad y eficiencia de sus procesos. Lo antes dicho, discrepa con la opción de enterrar los residuos sólidos vegetales recomendada por López, J. y otros (2017). para AGRíCOLA DEL CHIRA S.A. pues existen soluciones más eficientes, con mayores beneficios tanto para la empresa como para la sociedad. Téngase en cuenta que enterrar los residuos sólidos, también es un gasto para la empresa. Incluso, en algunos ingenios (como en LAREDO) se ha descartado la opción de enterrar la broza de este residuo vegetal.

En el caso de las empresas azucareras en el distrito de La Huaca, si existiese una Planta de Valorización de residuos que requiera broza de caña (una planta de biogás por ejemplo) se solucionaría el problema de AGRÍCOLA DEL CHIRA S.A., y se generaría el incentivo para que AGROAURORA S.A.C., siga este mismo camino.

Por lo expuesto, la regulación ambiental que se necesitaría principalmente sería una asociada al fomento de la valorización de residuos sólidos, para la disminución de 
quema de caña de azúcar. También se señala que, los resultados cualitativos denotan que si bien puede existir una regulación del tipo comando y control, esta debe ser complementada necesariamente por regulación de fomento de valorización de residuos vegetales del cultivo de caña. Lo señalado en el párrafo anterior, encuentra relación inmediata nuevamente con los preceptos del Análisis de Impacto Regulatorio (AIR), pues por lo general en los países existen "sistemas híbridos", es decir se cuenta con una combinación de regulación obligacional, sancionatoria y a su vez, regulación de fomento e incentivo, para atender problemáticas sociales

De esta manera, la Municipalidad Distrital de La Huaca resultaría la competente para gestionar el proyecto de una planta de valorización de residuos sólidos ${ }^{4}$, ejerciendo de manera adecuada y armónica su competencia de regular la emanación de humos y gases en su jurisdicción, de acuerdo a lo establecido en el artículo 80 de la LOM, sin afectar los compromisos establecidos en los EIA'S aprobados por el Gobierno Central a AGRÍCOLA DEL CHIRA y AGROAURORA, en el marco de la Ley del SEIA. Este proceder de la Municipalidad, sería una "regulación estratégica" y además "legalmente posible".

En todo caso, si las empresas cañeras en La Huaca no se acogen o son renuentes a participar de la regulación de fomento o incentivo, el OEFA podría desplegar o hacer uso de medidas sancionadoras o correctivas que se encuentren establecidas, en caso corresponda, de acuerdo a lo establecido en la Ley del SINEFA, pudiendo incluso ordenar la paralización de la quema de caña, en cuanto se logre acreditar un inminente riesgo o afectación propiamente al componente aire y/o salud de las personas.

Por otro lado, los expertos informantes coinciden en que sería un error pensar que los plazos de adecuación que se han dado en otros países a los ingenios azucareros

\footnotetext{
4 ARTícUlo 80.- SANEAMIENTO, SALUBRIDAD Y SALUD Las municipalidades, en materia de saneamiento, salubridad y salud, ejercen las siguientes funciones: (...) 4. Funciones específicas compartidas de las municipalidades distritales: 4.1 Administrar y reglamentar, directamente o por concesión el servicio de agua potable,

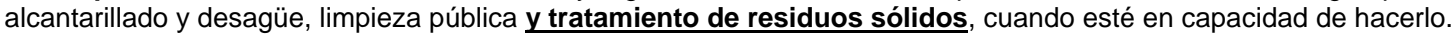
(...).
} 
para que dejen de quemar caña (entre 10 a 30 años), se puedan establecer en el Perú sin mayor análisis o reflexión.

En todo caso, se debería establecer acciones, metas y objetivos que se quieren lograr con el establecimiento del plazo. Asimismo, cada cierto período proceder a evaluar el avance de la meta. También, el plazo debe ser establecido con un criterio técnico o científico.

Sin embargo, el hecho de establecer plazos de adecuación de 10, 20 o 30 años, generaría que las empresas cañeras únicamente se esfuercen para lograr la meta legal. Por ejemplo, si legalmente o mediante el EIA, el Estado ordenó a AGROAURORA dejar de quemar caña en 30 años, no existen incentivos para que la empresa aplique sus máximos esfuerzos para dejar de quemar en menos tiempo

Por el contrario, si existiese una planta de valorización en la cual se gestione la broza de caña, esto sería un incentivo para el titular cañero para ejecutar sus máximos esfuerzos para aplicar cosecha mecanizada en verde, pues la broza que se genera por esta ya no sería un problema, sino, incluso un subproducto con valor económico. Al aplicar el titular cañero sus máximos esfuerzos, es probable que logre el resultado de reducción de quema en menor tiempo a lo que pudiese establecer una norma (10, 20 o 30 años).

\section{CONCLUSIONES}

En la actualidad, la quema de caña de azúcar es una actividad riesgosa, que genera externalidades negativas y que se encuentra desregulada; ello de conformidad con los resultados obtenidos en la investigación: Se necesita mayor regulación ambiental para que la quema de caña de azúcar disminuya.

La investigación arroja que es necesaria una regulación estratégica para la reducción de la quema de caña de azúcar en el distrito de La Huaca. Se debe dar preferencia a una regulación consistente con el fomento para la instalación de una planta de 
Una propuesta de regulación estratégica para el problema de la quema de caña de azúcar en el distrito de La Huaca, Piura, 2015 - 2018.

valorización de residuos sólidos, en la cual se reutilice de manera eficiente la broza de caña, cuya disposición constituye un problema de las empresas cañeras y la principal razón por la cual se ejecutan quemas en el distrito de La Huaca.

En referencia a la conclusión anterior, se recomienda a la Municipalidad Distrital de La Huaca, gestionar y articular con PROINVERSIÓN o un organismo de cooperación internacional y con otras entidades del sector público correspondiente, la implementación de un proyecto de planta de valorización de residuos (para generar energía eléctrica y/o biogás de preferencia) la cual remplace el botadero municipal actual, mediante una Asociación Público - Privada (APP) u obra pública, según corresponda.

Finalmente, si bien se recomienda la priorización de la construcción de una planta de valorización de residuos sólidos para atender la problemática de la quema de caña de azúcar en La Huaca, el Estado -el OEFA principalmente- debe de igual manera tener a su disposición otros mecanismos regulatorios (normas que regulen la actividad de quema de caña, normas sancionatorias, fortalecimiento del enforcement, etc.) que podría aplicar de acuerdo a las circunstancias y según sea necesario.

\section{REFERENCIAS BIBLIOGRÁFICAS}

Aguilar, A., Pérez, A., \& Aguilar, D. (2016). Nuevos paradigmas en la cosecha de la caña para el uso sustentable de toda la biomasa en las bioeléctricas. Parte I. ICIDCA. Sobre los Derivados de la Caña de Azúcar, 3-8 Obtenido de https://www.redalyc.org/pdf/2231/223152661001.pdf.

Alva, M. (2018). Las externalidades negativas y el impuesto selectivo al consumo. Obtenido de http://blog.pucp.edu.pe/blog/blogdemarioalva/2018/05/01/lasexternalidades-negativas-y-el-impuesto-selectivo-al-consumo/.

Gunningham, N., Grabosky, P., \& Darren, S. (1998). Smart Regulation: Designing Environmental Policy, Oxford: Clarendon Press. 
Hernandez, R., Fernandez, C., \& Baptista, M. (2014). Metodologia de la investigacion (6 ed.). Mexico: Mc Graw Hill education.

Hernández, R., \& Mendoza, C. (2018). Metodología de la investigación: las rutas cuantitativa, cualitativa y mixta . México: Mc Graw Hill Interamericana Editores, S.A. de C.V.

López, J. y otros (2017). Diagnóstico Operativo Empresarial - Agrícola del Chira S.A. (Tesis para obtener el grado de Magíster en Administración Estratégica de Empresas otorgado por la Pontificia Universidad Católica del Perú), Lima, Perú: Pontificia Universidad Católica del Perú.

Organismo Supervisor de la Inversión en Energía y Minería - OSINERGMIN. (2016). "Guía de Política Regulatoria N¹: Guía Metodológica para la realización de Análisis de Impacto Regulatorio en Osinergmin", Lima: Impresso Grafica S.A.

Villalobos, M. (2017). Influencia de la quema de biomasa de caña de azúcar en la concentración de PM2.5 en el aire de la zona urbana de Laredo. (Tesis para optar el titulo de ingeniero ambiental), Trujillo, Perú: Universidad Cesar Vallejo. 